\title{
RESONANCE INDUCED IMPEDANCE SENSING OF HUMAN BLOOD CELLS
}

\author{
Siyang Zheng, Mandheerej S. Nandra, Victor Shih, Wei Li and Yu-Chong Tai \\ Department of Electrical Engineering, California Institute of Technology, Pasadena, CA, U.S.A. \\ (Tel : +1-626-395-2227; E-mail: siyang@mems.caltech.edu)
}

\begin{abstract}
A challenging problem in AC impedance sensing of particles (e.g., blood cells in plasma) with micro electrodes is that with the shrinking of electrode surface area the electrode double layer capacitance decreases. Combined with the parallel stray capacitance, the system impedance is dominated by these capacitive components. Hence the sensitivity for particle sensing decreases. In this paper, we propose a new approach to solve the problem. The idea is to use resonant sensing by connecting an external parallel inductor to the system. At the resonant frequency, the capacitive components in the system were nullified by the inductor, leaving the electrolyte and particle impedance to be a major component in the system impedance. We then successfully demonstrated this idea by sensing $5 \mu \mathrm{m}$ polystyrene beads. More important, this technique was extended to sensing blood cells in diluted human whole blood and leukocyte rich plasma. The measured signal pulse height histogram matched well with known volume distribution of erythrocytes and leukocytes.
\end{abstract}

Keywords: Impedance, Resonance, Blood, Differentiation.

\section{INTRODUCTION}

Electrical impedance sensing has been used to measure biological materials, such as tissue samples and cell suspensions for over a hundred years [1, 2]. It has been used in bulk hemacytometers and flow cytometers extensively. The direct current (DC) resistive sensing extends to alternating current (AC) impedance sensing [3]. At low AC frequency (under $100 \mathrm{kHz}$ ), the signal is determined mainly by the cell volume. At higher frequency $(100 \mathrm{kHz}$ to $10 \mathrm{MHz})$, the intracellular structures also contribute to the overall measured impedance and become explorable measurands [4].

A serious problem in AC impedance sensing of particles (e.g., blood cells in plasma) with micro electrodes is that with the shrinking of electrode surface area the electrode double layer capacitance decreases. The double layer capacitance is in series with the channel impedance to be measured and it dominates the system impedance in low frequency range. In high frequency, the stray capacitance, which is in parallel with the channel impedance and caused by non-ideal electrode to electrode isolation, becomes the dominant part in system impedance. So to study particles with $\mathrm{AC}$ impedance sensing, the device is limited to a frequency range, which is high enough to bypass electrode double layer impedance and low enough that the stray capacitance does not play a significant role in overall system impedance. As the downsizing of the electrodes, the frequency range dominated by the double layer capacitance expands to higher frequency, hence the sensitivity for particle sensing decreases.

In this paper, we proposed a new approach to solve the problem. The idea was to use resonant sensing by connecting an external parallel inductor to the system. At the resonant frequency, the inherent capacitive component in the system was nullified by the inductor, leaving the channel impedance (composed of electrolyte and particle impedance) dominates in the system. The resonant excitation frequency could be selected by changing the inductance value. This work then validated this idea by sensing $5 \mu \mathrm{m}$ polystyrene bead. More important, this technique was explored successfully to sens e blood cells in diluted human whole blood and leukocyte rich plasma, which enabled us to directly measure the histogram of the contained cells. The results matched well with known volume histogram of erythrocytes and leukocytes.

\section{PRINCIPLE}

The principle of impedance particle sensing 
relies on the change of electrical impedance as particle replaces electrolyte in the sensing zone. This impedance change was measured by a pair of electrodes separated by an aperture. Ideally the channel impedance (which is represented by channel resistance $R_{S}$ in low frequency) should be the dominant impedance of the system so that the signal will be sensitive to the particle's existence. But for micro electrodes, the double layer surface capacitance $\mathrm{C}_{\mathrm{d} l}$ typically dominates the system impedance in low frequency and the stray capacitance $\mathrm{C}_{\text {st }}$ dominates in high frequency. As a result, the frequency spectra of air, DI water and electrolyte PBS inside device could not be distinguished (Fig.1). However, by having a parallel inductor to nullify the system capacitance components at the resonant frequency, a system with single resonant frequency was created as in SPICE simulation (Fig.2), and was confirmed with measurement (Fig.1). This way, the system total impedance was now most sensitive to the channel impedance change at the resonant frequency.

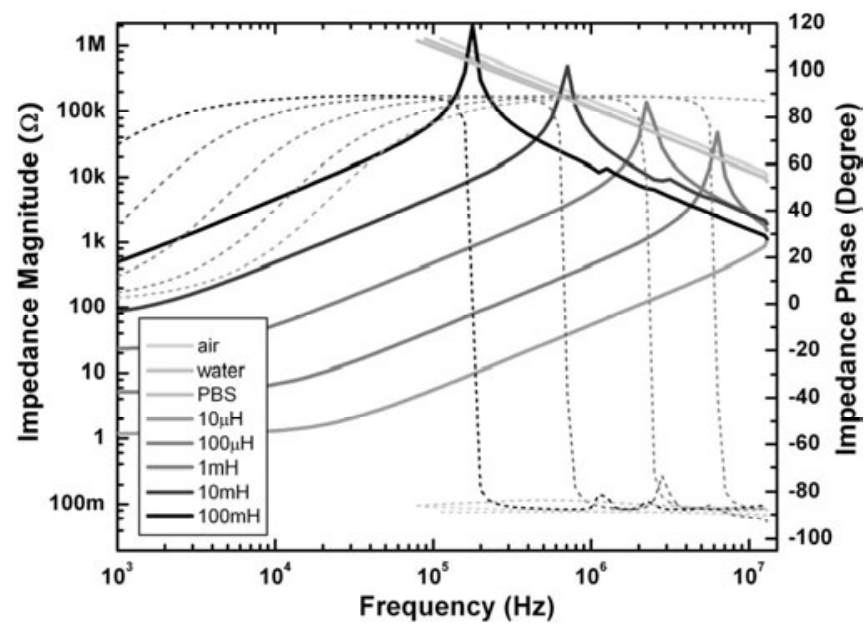

Fig. 1 Measured impedance magnitude response (solid lines) and phase response (dot lines) in different environments and with parallel inductors of different values in PBS.

\section{FABRICATION}

A device was made by bonding PDMS defined channels to glass with $\mathrm{Ti} / \mathrm{Pt}$ patterned electrodes (Fig.3). Two parallel sensing zones double the system throughput and enable differential input to sensing circuitry to reduce drift and noise. The two fluidic chambers were separated by an aperture. The width of the aperture was $14 \mu \mathrm{m}$. The length of the aperture was $20 \mu \mathrm{m}$. One pair of metal electrodes was used to sense the electrical impedance across one aperture. The size of the chamber was very large compared with the aperture so that the measured channel impedance was dominated by the impedance of the aperture region. The distance of the electrodes was $50 \mu \mathrm{m}$. At the inlet, some filter structures were designed to mechanically block contaminants and particle aggregates and prevent them from clogging the aperture. Channel height was optimized to be around $15 \mu \mathrm{m}$ so that all blood cells could pass while keeping signal magnitude as high as possible. In this study, conventional discrete coil inductors were used to demonstrate the principle, but the inductors can also be fabricated on the microchip to provide an integrated solution.[5] A lock-in amplification system with superior signal to noise ratio was used to track the system impedance change at resonant frequency.

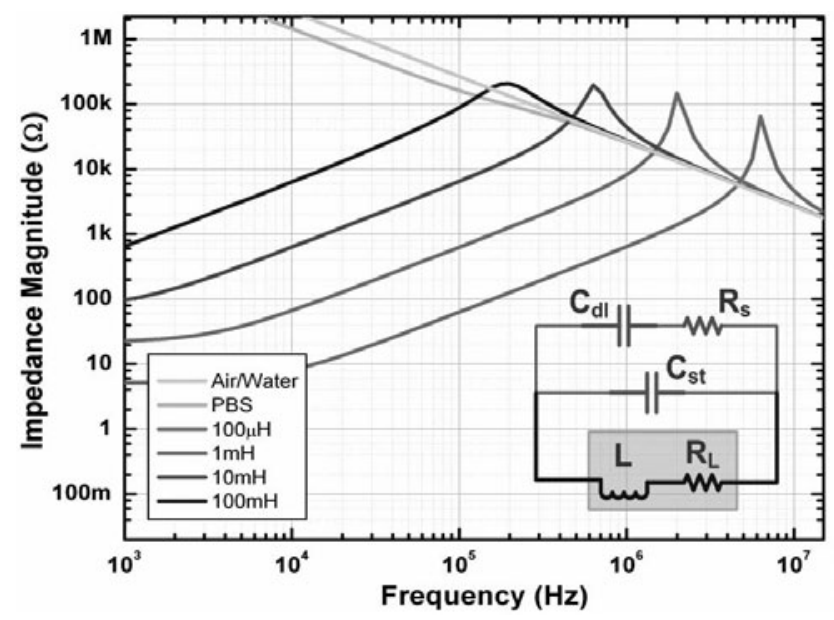

Fig. 2 SPICE circuit simulation of impedance magnitude response. Inset shows the equivalent circuit.

\section{RESULTS AND DISCUSSION}

Particle sensing was first validated with $5 \mu$ mdiameter polystyrene beads at resonant frequency of $104 \mathrm{kHz}$. The flow rate was $10 \mathrm{~nL} / \mathrm{min}$. The particle concentration was in the range of $104 / \mu \mathrm{L}$. Fig.4(a) shows an example of time trace of $5 \mu \mathrm{m}$ polystyrene beads. The peak height was $0.058 \mathrm{~V} \pm 0.013 \mathrm{~V}$ for a sample of 249 
beads in one testing. The duration of the peaks was $37.8 \mathrm{~ms} \pm 6.8 \mathrm{~ms}$, which corresponded well with the expected time that the beads passed the sensing zone. Fig.4(b) is a typical peak from the same data set. Polystyrene beads of $8 \mu \mathrm{m}$ and $10 \mu \mathrm{m}$ diameter were also tested and the signal magnitude was found to increase with the size of the beads.

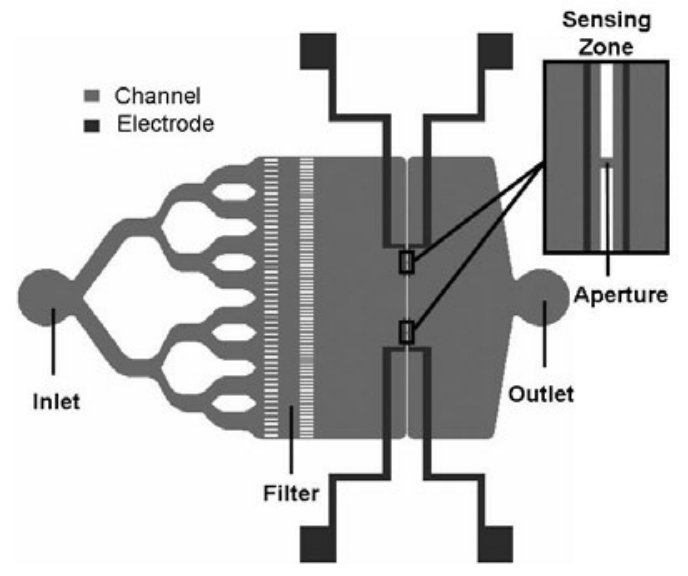

Fig. 3 Device layout.

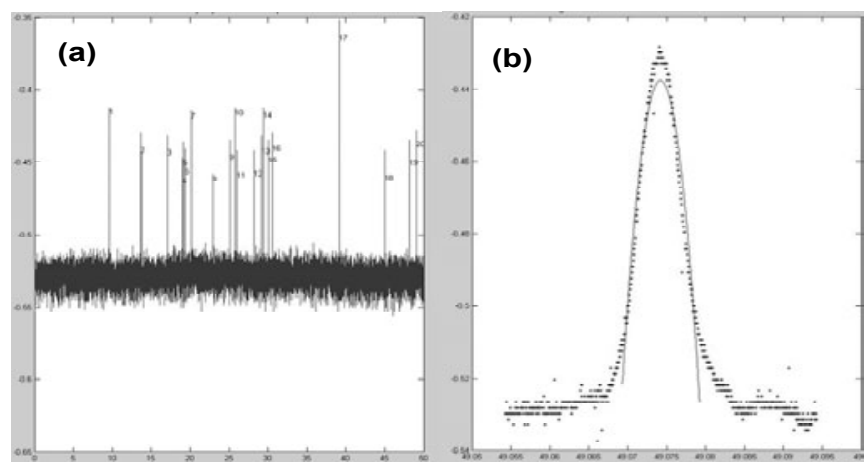

Fig. 4 Time trace (a) and an example peak (b) fro $m$ sensing of $5 \mu \mathrm{m}$ polystyrene beads at resonant fr equency $104 \mathrm{kHz}$.

Human blood cell sensing was performed either with diluted whole blood or diluted leukocyte rich plasma. Whole blood samples were obtained from healthy donors and used within 48 hours. Normal whole blood has an erythrocyte to leukocyte ratio about one thousand to one. So it is good for erythrocyte characterization without leukocyte interference. Leukocyte rich plasma was prepared with Wintrobe method. Erythrocyte to leukocyte ratio can be reduced by at least two orders of magnitude. So it is better for leukocyte testing.
Fig. 5(a) shows a time trace for human whole blood diluted by one thousand times. Close-up of one single peak was shown in Fig.5(b).

It's well known under DC and low frequency $\mathrm{AC}$ excitation, the change of channel impedance is correlated to the volume of the particles including biological cells. Based on our proposed system model and fitting parameters obtained from impedance spectra measurement, the volume of the particles was found be roughly linear with the change of total impedance magnitude at resonance, which was proportional to the peak height of the signal. So the distribution of impedance change can be used to measure the particle volume distribution.

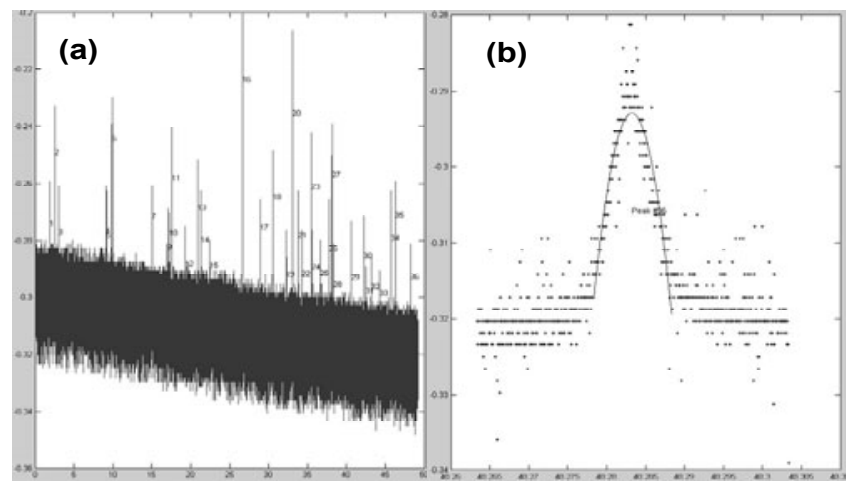

Fig. 5 Time trace (a) and an example peak (b) of $d$ iluted human whole blood testing at resonant freq uency $90.8 \mathrm{kHz}$.

For diluted whole blood, the peak height of blood cell traces had a wide distribution which indicates that the cell size varied. The histogram of pulse height matched well with the known volume distribution of erythrocytes (Fig.6). Leukocyte rich plasma was used for leukocyte sensing. The tail part of the peak height histogram was contributed mainly by leukocytes (Fig.7), while the small pulse height portion (under $0.1 \mathrm{~V}$ ) is presumably still dominated by erythrocytes. The tail part resembles the known leukocytes volume distribution.

\section{CONCLUSION}

We proposed a new approach to overcome the problem of small double layer in micro impedance sensing for particles in fluidic flow. A parallel 
inductor was introduced to induce system resonance. From impedance spectra measurement, one single peak was discovered for one inductance value. The inductor nullified capacitive components in the system at the resonant frequency. The devices were first tested with $5 \mu \mathrm{m}$ polystyrene beads. We then demonstrated human erythrocytes and leukocytes sensing with this approach. The histograms of the signal magnitude matched well with previous published volume distributions of the erythrocytes and leukocytes. The advantages of downsizing electrodes and the sensing zone in micro devices include increased sensitivity, lowered sample dilution factor and thus increased system throughput. The sensing frequency can be flexibly selected by changing the parallel inductance.

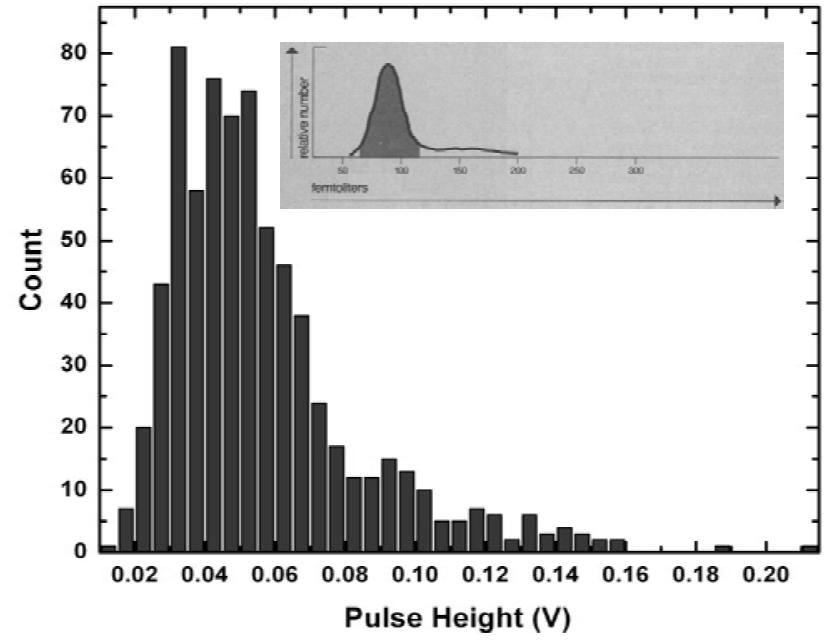

Fig. 6 Pulse height distribution of diluted human whole blood, in which leukocyte to erythrocyte ratio is about one to a thousand. Inset is erythrocyte volume histogram from [6].

\section{ACKNOWLEDGEMENTS}

The authors would like to thank for all the members at Caltech Micromachining Group for their value assistance. This work was supported by the National Space Biomedical Research Institute through NASA NCC 9-58.

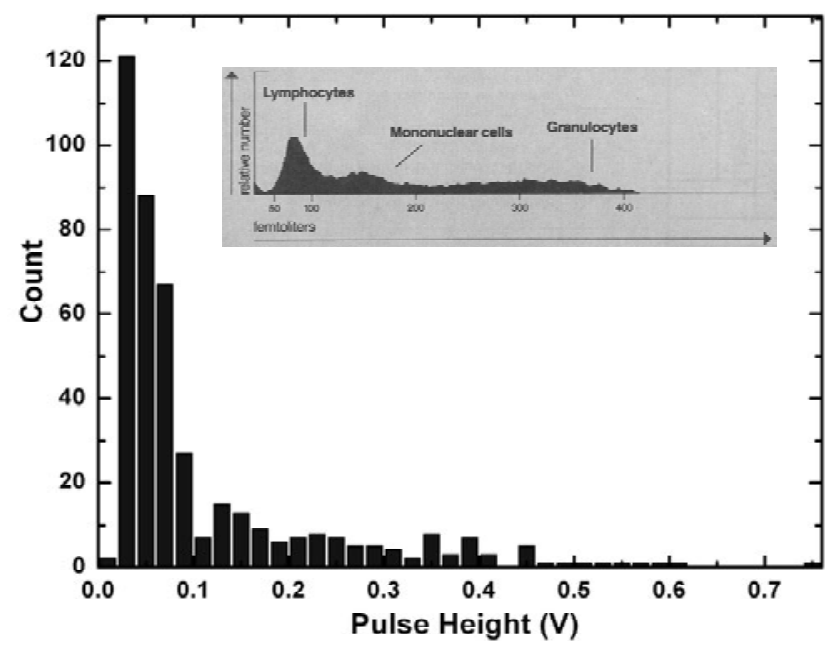

Fig. 7 Pulse height distribution of leukocyte rich plasma, in which leukocyte to erythrocyte ratio is about one to ten. Inset is leukocyte volume histogram [6].

\section{REFERENCES}

[1] O. F. Schanne and E. Ruiz P-Ceretti, Impedance Measurements in Biological Cells: John Wiley \& Sons, 1978.

[2] H. P. Schwan, "Linear And Nonlinear Electrode Polarization And Biological-Materials," Annals Of Biomedical Engineering, vol. 20, pp. 269-288, 1992.

[3] W. H. Coulter and W. R. Hogo, "Signal Modulated Apparatus for Generating and Detecting Resistive and Reactive Changes in a Modulated Current Path for Particle Classification and Analysis," U. S. P. Office, Ed. U.S.A., 1970.

[4] K. Asami, "Characterization of heterogeneous systems by dielectric spectroscopy," Progress In Polymer Science, vol. 27, pp. 1617-1659, 2002.

[5] W. Li, D. C. Rodger, E. Meng, J. D. Weiland, M. S. Humayun, and Y.-C. Tai, "Flexible parylene packaged intraocular coil for retinal prostheses," presented at the 4th International IEEE-EMBS Special Topic Conference on Microtechnologies in Medicine and Biology (MMB2006), Okinawa, Japan, 2006.

[6] S. B. McKenzie, Clinical Laboratory Hematology: Prentice Hall, 2004. 